\title{
Cluster magnetic fields from large scale structure shocks
}

\author{
Mikhail V. Medvedev*, Luis O. Silva ${ }^{\dagger}$ and Marc Kamionkowski** \\ ${ }^{*}$ Department of Physics and Astronomy, University of Kansas, KS 66045 \\ ${ }^{\dagger}$ GoLP/Centro de Fisica de Plasmas, Instituto Superior Técnico, 1049-001 Lisboa, Portugal \\ ${ }^{* *}$ California Institute of Technology, Mail Code 130-33, Pasadena, CA 91125
}

\begin{abstract}
The origin of the micro-Gauss magnetic fields in galaxy clusters is one of the outstanding problem of modern cosmology. We suggest that these fields could have been produced via the Weibel instability operating at shocks during the Large-Scale Structure formation and accretion shocks in Galaxy Clusters. We have performed three-dimensional particle-in-cell simulations of the nonrelativistic Weibel instability in an electron-proton plasma (with the ion-to-electron mass ratio of 100), in conditions typical of cosmological shocks. These simulations indicate that cluster fields could have been produced by shocks propagating through the intergalactic medium during the formation of large-scale structure or by shocks within the cluster. The strengths of the shockgenerated fields range from tens of nano-Gauss in the intercluster medium to a few micro-Gauss inside galaxy clusters. We discuss whether and how our results may change with the change the mass ratio to the realistic value of 1836 . We stress that even if the Weibel-generated small-scale magnetic fields decay with time, they can serve as seed fields that can be further amplified and inverse-cascade to larger scales by turbulent motions of post-shock MHD turbulence.
\end{abstract}

Keywords: magnetic fields — large-scale structure of universe — galaxies: clusters: general shock waves - plasmas - turbulence

PACS: $98.65 .-\mathrm{r}$, 95.30.Qd, 52.65.Rr, 52.35.Qz, 52.52.Tc

\section{INTRODUCTION}

The origin of the magnetic fields of 0.1 to few micro-Gauss strengths observed in galaxy clusters [1] poses one of the most intriguing problems in modern cosmology. The most common explanation invokes the amplification of seed or primordial fields by magnetohydrodynamic turbulence that have been excited during the processes of largescale-structure (LSS) formation. Although there are viable astrophysical mechanisms that can generate seed fields with $B \sim 10^{-16}$ Gauss or weaker [2], recent cosmological simulations [3] show that structure formation can amplify the field between the redshift of $z=50$ and present by a factor of 7000 in cluster centers and by a factor of 10-300 within filaments. Hence, in order to explain the observed intergalactic field, one needs seed fields as strong as $B \geq 10^{-10}$ Gauss.

Here we show that magnetic fields can be produced by collisionless shocks in galaxy clusters and in the intercluster medium (ICM) during LSS formation. Cosmological $\mathrm{N}$ body and hydrodynamic simulations of LSS formation $[4,5]$ have shown that shocks with Mach numbers up to $M \sim 100$ are ubiquitous on scales of few to few tens of megaparsecs. Theoretical analysis of non-magnetized collisionless shocks indicates that they can generate sub-equipartition fields [6]. We verify this prediction with state-ofthe-art numerical simulations. We present here three-dimensional (3D) particle-in-cell

CP932, Turbulence and Nonlinear Processes in Astrophysical Plasmas

$6^{\text {th }}$ Annual International Astrophysical Conference, edited by D. Shaikh and G. P. Zank

(C) 2007 American Institute of Physics 978-0-7354-0443-4/07/\$23.00 
(PIC) simulations of the nonrelativistic (with $v=0.1 c$ ) Weibel instability [7] in an electron-proton (with $m_{p} / m_{e}=100$ ) plasma, thus guaranteeing a clear separation of the relevant time scales. These simulations are computationally expensive compared to previous studies, which simulated relativistic shocks $(v \sim c)$ in an electron-positron or low-mass-ratio electron-ion plasmas $\left(m_{i} / m_{e} \leq 16\right)[8,9]$. Note that a recently discussed possibility that cluster shocks may produce the magnetic fields seen in galaxy clusters [10] was based on the assumption that the results of relativistic simulations will also apply in the nonrelativistic regime.

\section{THEORETICAL CONSIDERATION}

The mechanism of the field generation at shocks is rather simple [6]. As a shock propagates into an ambient medium, it reflects (or scatters) a fraction of the incoming (in the shock frame) particles back into the upstream region which then form counterpropagating streams. Both groups of particles (ICM/IGM and reflected particles) have bulk velocities of order the shock velocity $v_{\mathrm{sh}}$; they can also have some thermal spread. Both protons and electrons form the streams, so both species participate in the instability. One can consider each charged particle in these streams as an elementary current. Since like currents attract each other, it is energetically favorable for the elementary currents to merge into larger current filaments. This process is inhibited at scales smaller than the plasma skin depth, $\sim c / \omega_{p}$ ( $\omega_{p}$ is the plasma frequency), by strong electrostatic repulsion of like charges. In contrast, on large scales, the currents are quasi-neutral because of Debye shielding in a plasma. Hence, the filaments and associated magnetic fields grow rapidly. The process stops when most of the particles become trapped in the produced fields and can no longer amplify the field. This happens when the particle Larmor radius $\rho_{L}=v_{\perp B} / \omega_{c}\left(v_{\perp B}\right.$ is the particle velocity component transverse to the local magnetic field, and $\omega_{c}=e B / m c$ is the cyclotron frequency) becomes comparable to (or less than) the characteristic correlation scale $\lambda_{B}$ of the field, $\rho_{L} / \lambda_{B} \sim 1$. At this time, the particle distribution is effectively isotropized, and so $v_{\text {thermal }} \sim v_{\perp B} \sim v_{\text {sh }}$.

The anisotropy of the particle distribution near a shock can be parameterized as, $A=\left(\varepsilon_{\|}-\varepsilon_{\perp}\right) / \varepsilon_{\mathrm{tot}} \simeq\left(M^{2}-1\right) /\left(M^{2}+1\right)$, where $\varepsilon_{\|} \propto v_{\mathrm{sh}}^{2}$ is the energy of the particle along the shock propagation direction; $\varepsilon_{\perp} \propto v_{\text {thermal }}^{2} \simeq c_{s}^{2}$ is the thermal energy in the plane of the shock; $\varepsilon_{\text {tot }}=\varepsilon_{\|}+\varepsilon_{\perp}$ is the total energy; $c_{s}$ is the sound speed upstream; and the Mach number of the shock is $M=v_{\mathrm{sh}} / c_{s}$. For strong shocks, $M \gg 1$, the anisotropy parameter is close to unity, $A \sim 1$. At a shock, the bulk velocities of the electron and proton components are both comparable to the shock velocity. Hence, the protons dominate over the electrons in the overall energy budget, and the magnetic field generated by the electrons is negligible compared with that generated by the protons. The growth rate and the wavenumber of the fastest growing mode (which, in fact, sets the spatial correlation scale of the produced field) are $\gamma_{B}=A \omega_{p, p}\left(v_{\mathrm{sh}} / c\right)$ and $k_{B}=A \omega_{p, p} / c$, where $\omega_{p, p}=$ $\left(4 \pi e^{2} n_{p} / m_{p}\right)^{1 / 2} \approx 1.32 \times 10^{3} n_{p}^{1 / 2} \mathrm{~s}^{-1}$ is the proton plasma frequency, and $n_{p}$ and $m_{p}$ are the number density and the mass of the protons, respectively. (We use cgs units throughout, unless stated otherwise.) Order-of-magnitude estimates of the magneticfield $e$-folding time and the field correlation length at strong shocks $(M \gg 1)$ are read- 
ily obtained as $\tau_{B} \sim 1 / \gamma_{B} \simeq 2 \times 10^{2} v_{\mathrm{sh}, 7}^{-1} n_{\mathrm{ICM},-4}^{-1 / 2} \mathrm{~s}, \lambda_{B} \sim 2 \pi / k_{B} \simeq 10^{10} n_{\mathrm{ICM},-4}^{-1 / 2} \mathrm{~cm}$, for a typical ICM proton density of $n_{\mathrm{ICM}} \sim 10^{-4} \mathrm{~cm}^{-3}$ and a typical shock velocity $v_{\mathrm{sh}} \sim 10^{7} \mathrm{~cm} \mathrm{~s}^{-1}$; as usual, we denote $n_{\mathrm{ICM},-4}=n_{\mathrm{ICM}} /\left(10^{-4} \mathrm{~cm}^{-3}\right)$ and $v_{\mathrm{sh}, 7}=$ $v_{\mathrm{sh}} /\left(10^{7} \mathrm{~cm} \mathrm{~s}^{-1}\right)$. Since it takes $N \sim$ few $\times 10 e$-foldings to produce strong fields, we can readily estimate the thickness of a region of the field growth as $\Delta \sim N \tau_{B} v_{\mathrm{sh}} \sim N \lambda_{B}$.

The saturation level of the magnetic field is estimated from $\lambda_{B} \sim \rho_{L}=v_{\mathrm{sh}} / \omega_{c, p}$, where $\omega_{c, p}=e B / m_{p} c \approx 9.58 \times 10^{3} B \mathrm{~s}^{-1}$ is the proton cyclotron frequency. In a multiplespecies plasma, however, saturation occurs at equipartition with the lightest species [11]. To incorporate this, we introduce an efficiency factor $\eta$, which in the electron-proton plasma is of order $m_{e} / m_{p}$. Finally, $\varepsilon_{B}=\left[B^{2} / 8 \pi\right] /\left[m_{p} n_{p} v_{\mathrm{sh}}^{2} / 2\right] \simeq B^{2} /\left(8 \pi p_{\mathrm{sh}}\right) \simeq A^{2} \eta \sim$ $10^{-3}$, where $p_{\mathrm{sh}}$ is the gas pressure behind the shock, and the last estimate is for strong shocks, $A \sim 1$. Recently, it has been argued [14] that for nonrelativistic shocks with very small anisotropy of the particle distribution function (PDF), $A=T_{\perp} / T_{\|}-1 \leq m_{e} / m_{p}$, saturation occurs at $\varepsilon_{B} \sim\left(m_{e} / m_{p}\right)^{2}\left(T_{e} / T_{p}\right)^{2}$, which yields $\varepsilon_{B} \sim 10^{-6}$ for $T_{e} \sim T_{p}$, as is indicated by long-term PIC simulations (Spitkovski, private communication). This regime is not considered here.

Although there is no doubt that magnetic fields are generated at shocks through the Weibel instability, it is not clear whether they survive sufficiently far downstream to produce longstanding magnetic fields. The concern arises from the fact that the wavelength of the fastest-growing mode in the linear Weibel-instability analysis is very small, $\lambda_{B} \sim 2 \pi c / \omega_{p, p} \simeq 10^{10} \mathrm{~cm}$ for a typical ICM particle density of $n \sim 10^{-4} \mathrm{~cm}^{-3}$. Therefore, it is possible that the extremely short spatial scales-i.e., sharp field gradients—can be rapidly destroyed by dissipation on a plasma time scale of $\tau_{B} \sim 10^{2}$ s. Should this happen, the fields would occupy only a very narrow region near the shock front and, thus, would not result in long-lived cluster fields. Here two scenarios are possible. First, the correlation length (and, hence, the gradient scale) of the field increases with time, as PIC simulations indicate [12], thus drastically reducing diffusive (Ohmic) dissipation. Second, post-shock hydro/MHD turbulence can inverse-cascade the Weibel-generated "seed" field to larger scales by shearing motions of turbulent eddies.

\section{NONRELATIVISTIC 3D PIC SIMULATIONS}

In general, it is far from clear that nonrelativistic shocks can generate fields in the way relativistic shocks do. Hence, we have performed a set of simulations using 3D PIC code OSIRIS 2.0 [13]. In our PIC simulations, the initial conditions are taken to be two streams of electrons and ions moving with relative bulk velocity $v_{\text {sh }}$, which in our simulations we take to be $0.1 c$. The four "species" of particles (the upstream and downstream electrons and ions) is then each assigned a Maxwell-Boltzmann distribution of velocities about the bulk velocity. Our ions are "light protons," positively-charged particles with mass $m_{\text {ions }}=100 m_{e}$, a mass ratio large enough to guarantee that the electron and ion time scales are clearly separated. All of our simulations have volumes of $128^{3}$ cells, although the cell sizes differ.

We ran very long 3D simulations of colliding plasma slabs, for four sets of plasma parameters. One of the plasma slabs describes a shocked high-Mach-number plasma 
$\left(M=20, v_{\text {th,eshock }} / c=0.05\right.$, with either $v_{\text {th,ishock }} / c=0.005$ or $\left.v_{\text {th,i shock }} / c=0.0\right)$ with bulk motion along the $x_{1}$ direction. The different ion thermal velocities correspond to the two extreme cases of a strongly turbulent and a laminar shock. The second plasma slab describes the IGM/ICM plasma with either cold electrons, $T_{e}=0$, or hot electrons, $T_{e}=T_{i} \simeq 10 \mathrm{keV}$, such that $v_{\text {th,eIGM }} / c=0.05, v_{\text {th.iIGM }} / c=0.005$. All four of these simulations used a box of size $\left(25.6 \mathrm{c} / \omega_{p, e}\right)^{3}$. We found that the different physical parameters did not reveal any significant differences in the evolution of $\varepsilon_{B}$. Our present choice of simulation parameters is strongly limited by the time scales involved in the mechanisms described here, and it aims to illustrate the key features of the magneticfield generation in conditions relevant for nonrelativistic collisionless shocks.

It is important to note that the structure of a non-relativistic shock depends on its Mach number, not the shock speed itself. Hence, our simulations with $v_{\mathrm{sh}} / c \sim 0.1$ are re-scalable to any thermal velocity of a medium. Thus, for LSS filamets with $T \sim 10 \mathrm{eV}$ and $v_{\text {sh }} \sim 300-500 \mathrm{~km} \mathrm{~s}^{-1}$, our simulations are realistic.

In our study, we observe the key role played by the ions, with most of the magneticfield energy generated by the Weibel instability originating in the shocked ions. All the runs revealed $\varepsilon_{B} \simeq 10^{-3}$. Note that the Weibel-field growth of the ions saturates at lower relative $\varepsilon_{B}$ than for electrons $\varepsilon_{B, i} \sim\left(m_{e} / m_{i}\right)^{1 / 2} \varepsilon_{B, e}$, where for species $s=i, e$, $\varepsilon_{B, s}=\left(B^{2} / 8 \pi\right)\left(m_{s} n_{s} v_{\mathrm{sh}}^{2} / 2\right)^{-1}$. A strong thermalization between the electrons in the two slabs is achieved very early in time via the electron Weibel instability, but ion thermalization is not observed in our simulations. Other instabilities with longer time scales (e.g., the ion acoustic instability) will be responsible for this. These instabilities are not observed in our simulations since the simulation box is not large enough.

The time scale for energy transfer between the ions and the magnetic field is the time scale for the Weibel instability of the ions, longer than the electron Weibel instability by a factor of $\left(m_{i} / m_{e}\right)^{1 / 2}$, thus making its observation in numerical simulations very time consuming. The structure of the generated magnetic field depicted in Figure 1 shows the typical configuration of a Weibel-driven field in 3D and in the 2D plane transverse to the bulk motion of the shocked plasma, surrounding the self-generated current filaments, which are already evolving to longer wavelengths.

\section{DISCUSSION}

We have demonstrated that magnetic fields are produced at nonrelativistic collisionless shocks and their strengths are comparable to that observed in clusters. It is thus natural to explain the observed fields by the Weibel instability. If so, then the magnetization of clusters should begin around the reionization epoch, at redshifts of $z \sim 10-20$, when the gas becomes highly ionized and particle collisions become rare and inefficient. Our studies reveal that the magnetic field grows to an energy density of roughly a tenth of a percent of the initial kinetic-energy density, and hence constitutes a similar fraction, $\varepsilon_{B} \sim 10^{-3}$, of the thermal energy density of the shocked gas. The actual number depends on complicated nonlinear dynamics of the currents in the downstream region. This value of the equipartition parameter corresponds to a magnetic-field strength of order

$$
B \sim 10^{-8} \varepsilon_{B,-3}^{1 / 2} v_{\mathrm{sh}, 7} n_{\mathrm{ICM},-4}^{1 / 2} \text { Gauss. }
$$




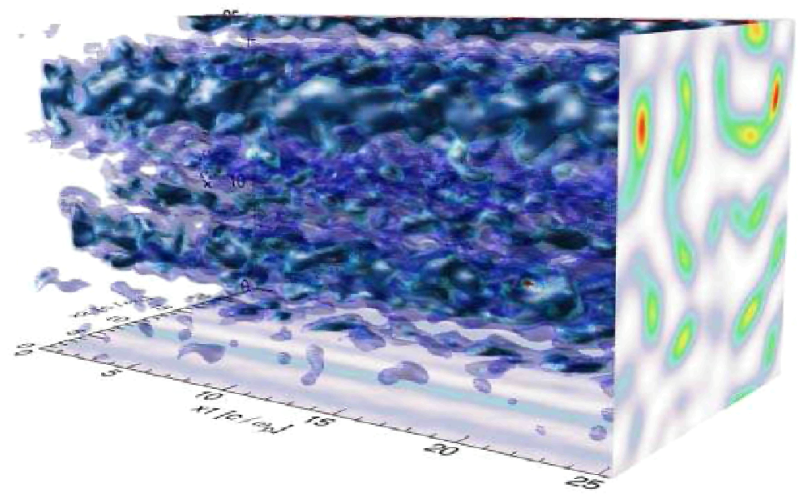

FIGURE 1. Magnetic field energy density at $t=2000 / \omega_{p, e}\left(\omega_{p, e}\right.$ is the electron plasma frequency). The blue iso-surfaces correspond to a value of $\varepsilon_{B} \simeq 8 \times 10^{-3}$. The projection in the $x_{2}-x_{3}$ plane (the shock plane) is the value of $\varepsilon_{B}$ averaged along $x_{1}$ (the shock propagation direction) with red color corresponding to a peak value of $\varepsilon_{B} \simeq 6 \times 10^{-2}$. The color scale in the projection plane is linear.

These values correspond to 1 to 10 nano-Gauss in the ICM (for typical $v_{\mathrm{sh}} \sim 300 \mathrm{~km} \mathrm{~s}^{-1}$ and $n \sim 10^{-6}-10^{-4} \mathrm{~cm}^{-3}$, respectively) and to 0.1 to 1 micro-Gauss inside galaxy clusters (for typical $v_{\mathrm{sh}} \sim 3000 \mathrm{~km} \mathrm{~s}^{-1}$ and $n \sim 10^{-4}-10^{-2} \mathrm{~cm}^{-3}$, respectively). Fields as high as few micro-Gauss can result from further amplification by turbulent motions in the IGM and by compression as a result of gas infall.

The simulations presented here model a strong shock with Mach number, $M=20$. Quite possibly, the predictions of [14] are relevant in this regime. The study specifically considers saturation of Weibel fields for very low PDF anisotropy, which could occur in weak shocks. Then, one can expect $\varepsilon_{B} \sim 10^{-6}$ or lower in this case.

LSS shocks can be observed via (i) synchrotron emission by the shock-accelerated electrons in the in situ generated magnetic fields; (ii) inverse-Compton scattering of cosmic microwave background photons by the shock-accelerated electrons; (iii) SunyaevZeldovich effect on thermal electrons in the shocked medium downstream; and/or (iv) an abrupt change in the Faraday rotation measure across the shock. The shock front appears to be very thin and will likely be unresolved. Since no sign of proton thermalization is seen by the end of the simulations, $t \sim 500 \omega_{p, p}$, we can put a constraint on the shock thickness,

$$
\Delta_{\mathrm{sh}}>10^{13} n_{\mathrm{ICM},-4}^{-1 / 2} \mathrm{~cm} \text {. }
$$

Our present analysis does not consider the evolution of the fields on cosmological time scales. There is a concern that the generated fields are very small-scale and can rapidly decay unless they inverse-cascade to larger (cosmological) scales, as suggested in [12]. Alternatively, even if they do not cascade by themselves, these fields can serve as "seed fields" to be further stretched by turbulent larger-scale motions of intra- and inter-cluster gas observed in cosmological large-scale structure simulations. 


\section{ACKNOWLEDGMENTS}

The simulations were performed in the eXpp cluster at IST, Lisbon. The work of MVM has been supported by DoE grant DE-FG02-04ER54790, NASA grant NNG04GM41G. The work of LOS was partially supported by FCT (Portugal) through grants PDCT/FP/FAT/50190/2003 and POCI/FIS/55905/2004. MK was supported by DoE DEFG03-92-ER40701 and NASA NNG05GF69G.

\section{REFERENCES}

1. C. Vogt and T. A. Enßlin, Astron. \& Astrophys., 412, 373 (2003).

2. N. Y. Gnedin, A. Ferrara, and E. G. Zweibel, Astrophys. J. , 539, 505 (2000).

3. M. Brüggen, et al., Astrophys. J. Lett. (in press) astro-ph/0508231 (2005).

4. F. Miniati, et al. Astrophys. J., 542, 608 (2000)

5. D. Ryu, H. Kang, E. Hallman, and T. W. Jones, Astrophys. J., 593, 599 (2003).

6. M. V. Medvedev and A. Loeb, Astrophys. J., 526, 697 (1999).

7. E. S. Weibel, Phys. Rev. Lett. , 2, 83 (1959).

8. L. O. Silva, et al. Astrophys. J. Lett., 596, L121 (2003).

9. J. T. Frederiksen, et al. Astrophys. J. Lett., 608, L13 (2004).

10. Y. Fujita, and T. N. Kato,Monthly Not. R. Astron. Soc., (in press), astro-ph/0508589 (2005).

11. J. Wiersma, and A. Achterberg, Astron. \& Astrophys. , 428, 365 (2004).

12. M. V. Medvedev, et al. Astrophys. J. Lett., 618, L75 (2005).

13. R. A. Fonseca, et al. Lecture Notes in Computer Science, 2331, 342 (Springer-Verlag: Heidelberg) (2002).

14. C. Ren, E. Blackman, and W. Fong, Phys. Plasmas, 14, 012901 (2007). 